\title{
BestMasters
}


Springer awards „BestMasters“ to the best master's theses which have been completed at renowned Universities in Germany, Austria, and Switzerland.

The studies received highest marks and were recommended for publication by supervisors. They address current issues from various fields of research in natural sciences, psychology, technology, and economics.

The series addresses practitioners as well as scientists and, in particular, offers guidance for early stage researchers. 
Janne Marie Soetbeer

\section{Dynamical Decoupling in Distance Measurements by Double Electron- Electron Resonance}

望 Springer Spektrum 
Janne Marie Soetbeer

Zurich, Switzerland

\section{BestMasters}

ISBN 978-3-658-14669-6

ISBN 978-3-658-14670-2 (eBook)

DOI 10.1007/978-3-658-14670-2

Library of Congress Control Number: 2016943852

Springer Spektrum

(C) Springer Fachmedien Wiesbaden 2016

This work is subject to copyright. All rights are reserved by the Publisher, whether the whole or part of the material is concerned, specifically the rights of translation, reprinting, reuse of illustrations, recitation, broadcasting, reproduction on microfilms or in any other physical way, and transmission or information storage and retrieval, electronic adaptation, computer software, or by similar or dissimilar methodology now known or hereafter developed.

The use of general descriptive names, registered names, trademarks, service marks, etc. in this publication does not imply, even in the absence of a specific statement, that such names are exempt from the relevant protective laws and regulations and therefore free for general use.

The publisher, the authors and the editors are safe to assume that the advice and information in this book are believed to be true and accurate at the date of publication. Neither the publisher nor the authors or the editors give a warranty, express or implied, with respect to the material contained herein or for any errors or omissions that may have been made.

Printed on acid-free paper

This Springer Spektrum imprint is published by Springer Nature The registered company is Springer Fachmedien Wiesbaden GmbH 
"I am not aware of any other field of science outside of magnetic resonance that offers so much freedom and opportunities for a creative mind to invent and explore new experimental schemes (...)"

Richard R. Ernst, Nobel Lecture, December 9, 1992 


\section{Acknowledgements}

I would like to thank Yevhen Polyhach for the supervision of this master thesis. Thanks goes to Kamila Guerin for showing me the spin labelling technique for the T4 Lysozyme sample. This thesis would have not been possible without Andreas Dounas work on the optimisation of adiabatic inversion pulse envelopes. I am very much obliged to also thank Andrin Doll for his help at setting up all AWG measurements at both X and Q band. Finally, I would like to thank Professor Gunnar Jeschke for the opportunity to

carry out my master thesis in his group and for all the support and discussion related to this thesis. 


\section{Abstract}

The relaxation behaviour of model systems mimicking different spin-labelled electron spin environments have been studied including TEMPOL, spin-labelled T4 Lysozyme, spin-labelled WALP23 and rigid spin-labelled molecules. Carr-Purcell (CP) and Uhrig pulse sequences for a fixed total sequence time were used to record echo relaxation traces to find the optimal dynamical decoupling (DD) scheme which allows for efficient reduction of electron spin coherence losses for a given relaxation behaviour. Addition of $\pi$ refocusing pulses led to stronger nuclear modulation of the recorded relaxation traces and to signal contributions from both refocused and stimulated echos at the acquisition position. The latter complication was alleviated by small time shifts introduced into the pulse sequence.

The relaxation behaviour was characterised by phase relaxation time $T_{\mathrm{m}}$ and stretch parameter $\xi$ which were shown to both depend on the spin environment and the dominating relaxation mechanism. Deviations from literature values could be explained by the inherent nature of the relaxation measurement. DD schemes proofed most successful for $\xi>1$ as expected from theory. Overall, Uhrig schemes allowed for stronger suppression of coherence losses for interpulse delays at which the magnetisation in CP-type set ups had already decayed.

Based on the relaxation characterisation the DD schemes were extended to corresponding DD-double electron electron resonance (DEER) experiments by addition of pump pulses for a rigid biradical model system in $\mathrm{d}_{8}$-OTP and T4 Lysozyme in $\mathrm{H}_{2} \mathrm{O}-\mathrm{d}_{8}$ Glycerol mixture. CP-type DD observer pulse sequences of order $n=1,2$ and 4 were used to record DEER traces. The promising relaxation study results for Uhrig-type DD schemes did not translate into a superior Uhrig-DEER experiment relative to a CP 
derived set up for different reasons at $\mathrm{X}$ and $\mathrm{Q}$ band.

Signal artifacts related to $m$ times pump spin inversion with $1 \leq m<n$ in DD-DEER experiments of order $n=2$ or 4 were strongly reduced by increasing inversion efficiency based on pump pulse optimisation. Linear-chirp and asymmetric hyperbolic secant pulses were tested with optimal pulse parameters obtained from pulse simulation including resonator profile compensation. Measurements at $Q$ band showed pump pulse underperformance with respect to inversion efficiencies expected from theory. This resulted in strong contributions from signal artifacts in the corresponding DD-DEER traces. Nearly full suppression of the latter were achieved at $\mathrm{X}$ band with sacrifices in terms of sensitivity relative to $Q$ band.

X-band order $n=2$ DD-DEER traces for the low concentration rigid biradical sample allowed for direct DeerAnalysis fitting without data post processing. 


\section{Contents}

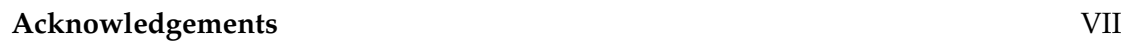

Abstract IX IX I

Glossary $\quad$ XV

List of Figures $\quad$ XIX

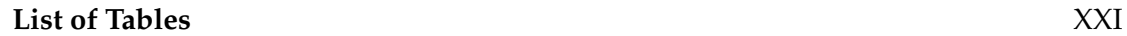

$1 \quad$ Introduction and Theory 1

\begin{tabular}{lll}
\hline .1 & Motivation of research & 1
\end{tabular}

$\begin{array}{lll}1.2 & \text { Spin Hamiltonian } & 2\end{array}$

1.2.1 Electron Zeeman interaction $\mid \hat{H}_{E Z} \ldots \ldots \ldots \ldots . \ldots \ldots$

1.2.2 Hyperfine interaction $\hat{H}_{H F} \ldots \ldots \ldots \ldots \ldots$. . . . . . . . . 4

1.2 .3 Electron-electron spin interactions $\hat{H}_{E X}$ and $\hat{H}_{D D} \ldots \ldots \ldots \ldots$

1.2.3.1 Exchange interaction $\hat{H}_{\mathrm{EX}} \ldots \ldots \ldots \ldots \ldots$

1.2.3.2 Dipole-dipole interaction $\hat{H}_{\mathrm{DD}} \ldots \ldots \ldots \ldots$

\begin{tabular}{lll}
\hline 1.3 Pulses in EPR experiments & 6
\end{tabular}

1.3.1 Magnetisation vector picture . . . . . . . . . . . . . . . . . 7

1.3 .2 Adiabatic pulses . . . . . . . . . . . . . . . . . . 8

1.3.2.1 Linear-chirp pulse . . . . . . . . . . . . . . . . . . . 10

1.3.2.2 Hyperbolic secant pulse . . . . . . . . . . . . . . . . . . 10

1.3.2.3 Hyperbolic secant pulse of order $h$. . . . . . . . . . . . . 10

1.3 .3 Inversion Characterisation . . . . . . . . . . . . . . . . . . . . 11

$\begin{array}{lll}1.4 \text { Pulsed EPR experiments } & 11\end{array}$

1.4.1 Primary and stimulated echo . . . . . . . . . . . . . . . . . . . 11

1.4 .2 Nutation experiment $\ldots \ldots \ldots \ldots$. . . . . . . . . . . . . . 13

1.4 .3 DEER sequences . . . . . . . . . . . . . . . . . 13

1.4.3.1 Computation of echo amplitude modulation function . . . . . . . 13 
1.4.3.2 4-pulse DEER sequence $\ldots \ldots \ldots \ldots$

1.4 .3 .3 5-pulse DEER sequence $\ldots \ldots \ldots \ldots \ldots \ldots$

1.4 .3 .4 Pump pulses in DEER $\ldots \ldots \ldots \ldots$

1.4.3.5 DEER signal . . . . . . . . . . . . . . . . . . . 20

1.4 .4 DEER data analysis . . . . . . . . . . . . . . . . . 20

$\begin{array}{ll}1.5 \text { Relaxation } & 21\end{array}$

1.5 .1 Relaxation processes in immobilised samples $\ldots \ldots \ldots \ldots . \ldots . . \ldots 22$

$\begin{array}{lll}1.6 & \text { Dynamical Decoupling } & 23\end{array}$

2 Material and Methods 25

$\begin{array}{ll}2.1 \text { Model systems } & 25\end{array}$

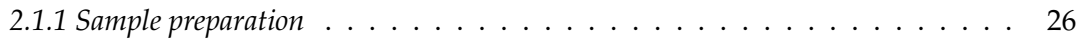

2.1.1.1 TEMPOD . . . . . . . . . . . . . . . . . . . . 27

2.1.1.2 Rigid radicals MS 107 and MSA $236 \ldots \ldots \ldots \ldots$

2.1.1.3 T4 Lysozyme. . . . . . . . . . . . . . . . . . . . . 27

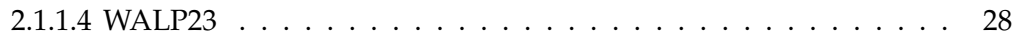

$\begin{array}{ll}2.2 \text { Instrumentation } 28 & 28\end{array}$

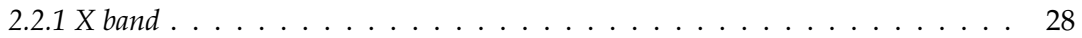

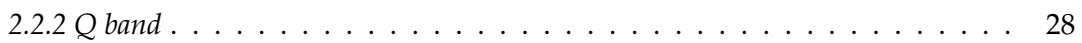

2.2 .2 .1 Home-built spectrometer $\ldots \ldots \ldots \ldots \ldots . \ldots \ldots$

2.2 .2 .2 Commercial . . . . . . . . . . . . . . . . . . . . 29

$\begin{array}{lll}2.3 & \text { Experiments } & 29\end{array}$

\begin{tabular}{|l|l|}
2.4 Relaxation Measurements & 29
\end{tabular}

2.4 .1 Pulse sequences . . . . . . . . . . . . . . . . . . . . . 30

2.5 DEER Measurements 32

2.5 .1 Pulse sequence. . . . . . . . . . . . . . . . . . . . . 32

$2.5 .1 .1 \times$ band $\ldots \ldots \ldots \ldots \ldots \ldots$

2.5 .1 .2 Qband . . . . . . . . . . . . . . . . . 39 
2.5 .2 Pump pulses . . . . . . . . . . . . . . . 40

2.5.2.1 Design . . . . . . . . . . . . . . . . 40

2.5 .3 Resonator compensation $\ldots \ldots \ldots \ldots \ldots \ldots \ldots \ldots$

2.5.3.1 Inversion characterisation . . . . . . . . . . . . . . . 41

2.5 .4 DEER signal analysis . . . . . . . . . . . . . . . . . . 42

3 Results and Discussion 43

$\begin{array}{lll}3.1 \text { Relaxation } & 43\end{array}$

3.1.1TEMPOL . . . . . . . . . . . . . . . . . . . 43

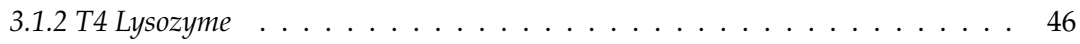

3.1 .3 WALP23 . . . . . . . . . . . . . . . . . . . . . 47

3.1.4 Comparison of free TEMPOL, spin-labelled T4 Lysozyme and WALP23 . . . . 48

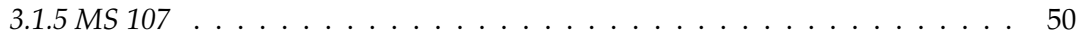

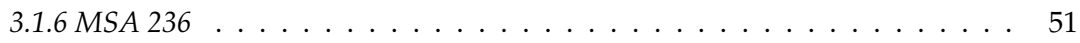

3.2 DEER experiments 53

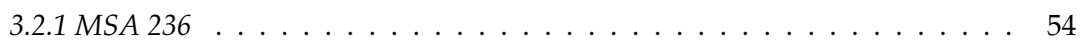

3.2.1.1 Q Band . . . . . . . . . . . . . . . . . 54

$3.2 .1 .2 \times$ band $\ldots \ldots \ldots \ldots \ldots \ldots \ldots \ldots \ldots$

3.2.1.3 DEER data analysis $\ldots \ldots \ldots \ldots$. . . . . . . . . . 60

3.2 .2 T4 Lysozyme in $\mathrm{H}_{2} \mathrm{O}-\mathrm{d}_{8} \mathrm{Gly} \ldots \ldots \ldots \ldots \ldots$. . . . . . . . . 62

3.2 .2 .1 Qband . . . . . . . . . . . . . . . . . 63

$3.2 .2 .2 \times$ band $\ldots \ldots \ldots \ldots \ldots \ldots \ldots \ldots \ldots \ldots$

3.2 .2 .3 DEER data analysis $\ldots \ldots \ldots \ldots \ldots 6$

3.2 .3 Comparison X-and Q-band DEER experiments. . . . . . . . . . 67

4 Conclusion and Outlook 69

\begin{tabular}{ll}
\hline Bibliography & 73
\end{tabular} 


\section{Glossary}

$A M(t) \quad$ Amplitude modulation function.

$B(t) \quad$ Intermolecular background function.

$B_{\text {eff }} \quad$ Effective field created by $B_{1}$ and $B_{0}$.

$D \quad$ Dimensionality of the homogeneous spin distribution.

$F(t) \quad$ Intramolecular form function.

$F M(t) \quad$ Frequency modulation function.

$I(f) \quad$ Inversion profile of pulse.

$K(t, r) \quad$ Kernel function.

$M_{0} \quad$ Magnetisation vector at equilibrium.

$M_{z} \quad$ Longitudinal magnetisation.

$N \quad$ Factor for extending 5-DEER experiment by $N \pi$ 5-DEER pulses.

$P(r) \quad$ Distance distribution.

$Q_{\text {min }} \quad$ Minimal adiabaticity factor at resonance.

$S(t) \quad$ Simulated signal.

$T_{1} \quad$ Longitudinal relaxation time.

$T_{2} \quad$ Transverse relaxation time.

$T_{\mathrm{m}} \quad$ Phase memory time.

$V(0) \quad$ Echo intensity at time zero.

$V(t) \quad$ Primary DEER trace.

$\Delta \quad$ Modulation depth.

$\Delta \nu \quad$ Total sweep width of chirp pulse.

$\Delta t \quad$ Time increment of interpulse delays.

$\Omega_{\mathrm{A}} \quad$ Frequency of the DEER observer pulses.

$\Omega_{\mathrm{B}} \quad$ Frequency of the DEER pump pulses.

$\Omega_{\mathrm{S}} \quad$ Resonance offset.

$\Phi_{0} \quad$ Ground state electronic wavefunction.

$\alpha \quad$ Regularisation parameter.

$\beta \quad$ Flip angle of an applied microwave pulse.

$\beta_{\mathrm{HS}} \quad$ Truncation factor of HS pulse.

$\delta T \quad$ Time shift to avoid observer and pump pulse overlap.

$\delta \tau \quad$ Fixed time shift to separate refocused and stimulated echo.

$\hat{H}_{0} \quad$ Spin Hamiltonian.

$\hat{H}_{\text {DD }} \quad$ Dipole-Dipole Hamiltonian.

$\hat{H}_{\text {EX }} \quad$ Exchange Hamiltonian. 
$\hat{H}_{\text {EZ }} \quad$ Electron Zeeman Hamiltonian.

$\hat{H}_{\mathrm{HF}} \quad$ Hyperfine Hamiltonian.

$\hat{H}_{\mathrm{NQ}} \quad$ Nucler Quadrupole Hamiltonian.

$\hat{H}_{\mathrm{NZ}} \quad$ Nuclear Zeeman Hamiltonian.

$\hat{H}_{\text {ZFS }} \quad$ Zero-Field Hamiltonian.

$\hat{\vec{I}} \quad$ Nuclear Spin vector operator.

$\hat{\vec{S}} \quad$ Electron spin vector operator.

$\hbar \quad$ Reduced Planck constant, $1.054571726(47) \cdot 10^{34} \mathrm{~J} \cdot \mathrm{s}$.

$\kappa \quad$ Normalised inversion quality of pulse.

$\lambda \quad$ Fraction of spins excited by pump pulse.

$\left\langle S_{\mathrm{A} y}\right\rangle \quad$ Expectation value of the $S_{\mathrm{A} y}$ operator corresponding to observable magnetisation.

$\mu \quad$ Macroscopic moment.

$\mu_{0} \quad$ Vacuum permeability.

$\mu_{\mathrm{B}}=\beta_{\mathrm{e}} \quad$ Bohr magneton.

$\nu_{1}(f) \quad$ Resonator profile.

$\nu_{\mathrm{DD}} \quad$ Dipole dipole coupling in frequency units.

$\nu_{\text {final }} \quad$ Final frequency for chirped pulse relative to observer frequency.

$\nu_{\text {initial }} \quad$ Starting frequency for chirp pulse relative to observer frequency.

$\nu_{\mathrm{mw}} \quad$ Frequency of the microwave radiation.

$\omega_{0} \quad$ Larmor frequency.

$\omega_{\text {eff }} \quad$ Effective frequency.

$\omega_{\mathrm{mw}} \quad$ Microwave frequency of applied pulse field.

$\rho \quad$ Spin density.

$\sigma_{\text {echo }} \quad$ Density operator at the echo position.

$\sigma_{\text {eq }} \quad$ Density operator at thermal equilibrium.

$\tau \quad$ Interpulse delay.

$\theta \quad$ Polar angle beween $\vec{B}_{0}$ and $\vec{r}$.

$\theta, \phi \quad$ Polar angles in PAS of $\mathrm{g}$.

$1 \quad$ Unit tensor.

$\underline{\text { A }} \quad$ Hyperfine tensor.

D Dipolar coupling tensor.

I Exchange coupling tensor.

$\mathrm{g} \quad \mathrm{g}$ tensor.

$\overrightarrow{1} \quad$ Unit vector.

$\vec{B} \quad$ External static magnetic field $\left(0,0, \mathrm{~B}_{0}\right)$.

$\vec{B}_{0} \quad$ Static magnetic field vector.

$\vec{B}_{0}^{T} \quad$ Transposed external magnetic field vector.

$\vec{B}_{1}(t) \quad$ Pulse field.

$\vec{r} \quad$ Spin-spin vector of length $\mathrm{r}$.

$\xi \quad$ Stretch parameter in relaxation law.

$a_{\mathrm{s}} \quad$ Pulse amplitude. 


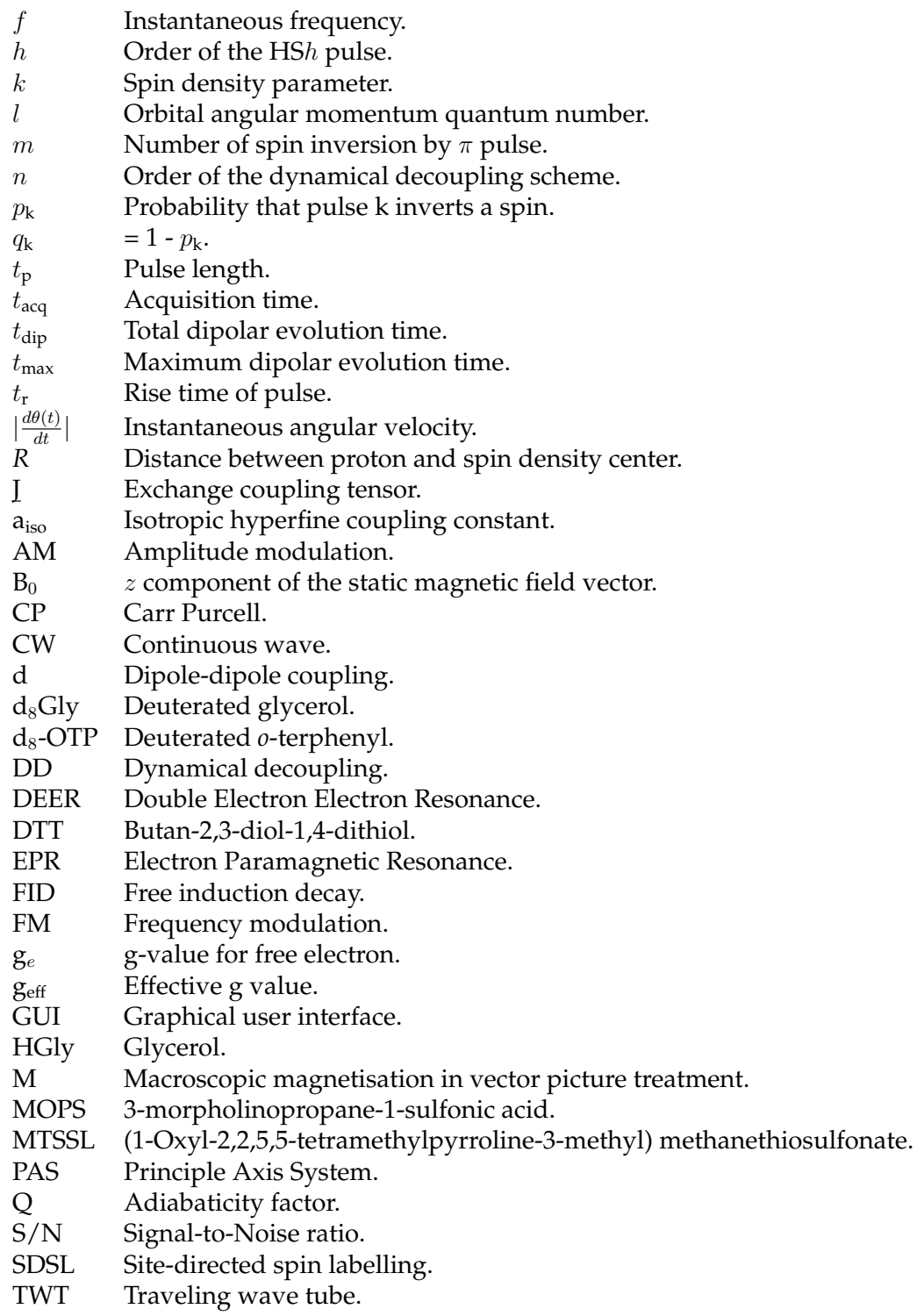




\section{List of Figures}

1 Primary and stimulated echo sequences. . . . . . . . . . . . . . . . . . 12

2 Pulse sequence for nutation experiment. . . . . . . . . . . . . . . . . . . . . . . . . . . . . . . 15

3 Comparison of the 4-DEER and 5-DEER sequences. . . . . . . . . . . . 15

4 Chemical structure of monoradical MS 107. . . . . . . . . . . . . . . 26

5 Pulse sequences for $T_{2^{-}}, 4,5,7-D E E R$ and 9-Uhrig relaxation measurements. 31

$6 \quad$ Stimulated and refocused echo positions in 4- and 5 DEER observer scheme. 32

$7 \quad$ Influence of stimulated echo contribution in rect-5' -DEER traces for MSA

236 at Qband. . . . . . . . . . . . . . . . . . . . 33

$8 \quad 5^{\prime}$-DEER and $9^{\prime}$-DEER pulse sequences. . . . . . . . . . . . . . . . . . . . . . . . . . . 34

$9 \quad 9^{\prime}$-Uhrig-DEER sequence. . . . . . . . . . . . . . . . . . 37

$10 \quad$ Influence of the acquisition time on fitting parameters $T_{\mathrm{m}}$ and $\xi$. . . . . . 45

11 Comparison of $T_{2}$ and DEER- $T_{2}$ measurements of 11 and $>75 \mu \mathrm{M}$ TEMPOL in $\mathrm{H}_{2} \mathrm{O}-\mathrm{HGly} . \ldots \ldots \ldots \ldots$. . . . . . . . . . . . . . . 46

12 Relaxation traces for $11 \mu \mathrm{M}$ TEMPOL in $\mathrm{H}_{2} \mathrm{O}-\mathrm{HGly}$ and $50 \mu \mathrm{M}$ WALP23-

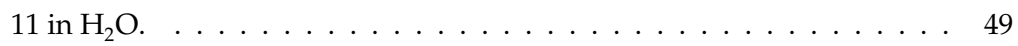

13 Relaxation traces for T4 Lysozyme in $\mathrm{H}_{2} \mathrm{O}-\mathrm{d}_{8}$ Gly at $\mathrm{Q}$ and X band. . . . . . 49

14 X-and Q-band relaxation traces for mono- and biradical MS 107 and MSA 236 in $\mathrm{d}_{8}$-OTP. . . . . . . . . . . . . . . . . 51

15 4-DEER signal analysis for the model sample MSA 236 using DeerAnaly-

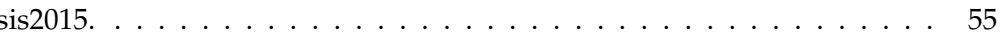

16 Q-band chirp-5'-DEER traces for MSA 236 in $\mathrm{d}_{8}$-OTP for three different

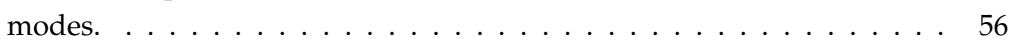

17 Q-band rect- and chirp-5'-DEER traces for MSA 236 in $\mathrm{d}_{8}$-OTP. . . . . . . 58

18 X-band rect-, chirp- and HSh-5'-DEER traces for MSA 236 in $\mathrm{d}_{8}$-OTP. . . 59

19 Tikhonov regularisation $(\alpha=0.1,1,10)$ for X-band HSh-4 and 5'-DEER of MSA 236. . . . . . . . . . . . . . . . . . 60

20 X-band asymmetric HSh-4- and 9'-DEER traces for MSA 236 in $\mathrm{d}_{8}$-OTP. .61

$21 \quad$ Q-band inversion efficiency assessment by $\mathrm{S}_{\mathrm{z}}$ for chirp and HSh. . . . . . 64

22 Q-band rect-4 and HSh 4- and 5'-DEER traces for T4 Lysozyme in $\mathrm{H}_{2} \mathrm{O}$ -

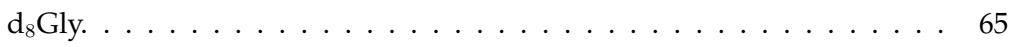

23 X-band HSh-4 and 5'-DEER traces for T4 Lysozyme in $\mathrm{H}_{2} \mathrm{O}-\mathrm{d}_{8} \mathrm{Gly}$. . . . . 66

24 HSh-5'-DEER derived $P(r)$ comparison for T4 Lysozyme in $\mathrm{H}_{2} \mathrm{O}_{-} \mathrm{d}_{8}$ Gly at $X$ and $Q$ band. . . . . . . . . . . . . . . . 68 


\section{List of Tables}

$1 \quad$ Fit parameter $T_{\mathrm{m}}$ and $\xi$ for TEMPOL 4-DEER relaxation measurements. . $\quad 44$

2 Fit parameter $T_{\mathrm{m}}$ and $\xi$ TEMPOL in $1: 1 \mathrm{H}_{2} \mathrm{O}$-HGly relaxation measurements. 46

3 Fit parameter $T_{\mathrm{m}}$ and $\xi$ for T4 Lysozyme-72-131 4-DEER relaxation mea-

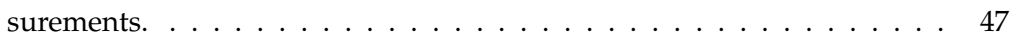

$4 \quad$ Fit parameter $T_{\mathrm{m}}$ and $\xi$ for WALP23 4-DEER relaxation measurements. . $\quad 48$ 